\title{
Transport properties of the hierarchical model for stretched polymers
}

\author{
Chen-Ping Zhu ${ }^{1,2}$, Shi-Jie Xiong ${ }^{1, *}$ and Tao Chen ${ }^{1}$ \\ 1 National Laboratory of Solid State Microstructures and Department of Physics, Nanjing University, Nanjing 210093, \\ People's Republic of China \\ ${ }^{2}$ College of Science, Nanjing University of Aeronautics and Astronautics, Nanjing 210016, People's Republic of China.
}

\begin{abstract}
We prove that the hierarchical fractal model recently proposed for describing the stretched polymers [A. N. Samukhin et al, Phys. Rev. Lett. 78, 326(1997)] is equivalent to a one-dimensional chain with hierarchical aperiodic structure. By use of the transfer matrix technique we calculate the electronic transmission and the dc conductance. We find that there exist sharp-edged transmission subbands and gaps, but the transmission subbands are rich in substructures which show self-similarity. The temperature dependence of conductance $g(T)$ is sensitive to the variation of the Fermi level and to the structure parameters $m$ and $n$ of the original fractal structure. The relationship of the obtained results to the experimental data and other theoretical works is discussed.
\end{abstract}

PACS numbers: 73.61.Ph,05.60.+w,71.30.+h,72.90.+y

*E-mail: sjxiong@nju.edu.cn

\section{INTRODUCTION}

It is widely accepted that polymers represent complicated structures which consist of coupled one-dimensional (1D) chains, and many unusual properties can be understood based on their quasi-1D feature. During last decades analytical and numerical studies revealed that the phenomenon of Anderson localization is a characteristic feature of 1D systems. For a quasi-1D system the interchain coupling tends to delocalize the electronic states, which may lead to extended states when interchain diffusion rate $\omega$ exceeds a threshold value $\left.\omega_{c}\right]$. Moreover, conductance of several polymers, such as stretched polyacetyllene and polyanilline, is enhanced by a few orders of magnitude upon doping and can even reach high valuestypical of metals, while the temperature dependence of static conductance $\left(g_{d c}\right)$ widely vary from sample to sample 3 目.

In order to understand the effect of chain-windings and random interchain couplings on the electronic transport properties in quasi-1D polymers, Xiong et a $\mathbf{6} 10$ have investigated the transmission coefficient and conductance for models of winding and randomly coupled polymers. It is proved that for such systems there exist conducting subbands with "dilute" structure which can account for the high values of conductance and its insulator-like temperature dependence of some polymers. On the other hand, Samukhin et. a 11 suggested that the peculiar features of conducting polymers can be derived based on a special hierarchical structure-a nearly 1D fractal.

In this paper we investigate the transmission spectrum of the hierarchical structure proposed in 11 . We prove that this fractal network is equivalent to a 1D chain with hierarchical aperiodic structure for the transport of electrons in the stretched direction. By solving the Schrödinger equation of such an effective 1D model by the standard transfer matrix technique, we obtain the transmission coefficient as a function of energy for different stages (or size) of the structure. We find that there also exist "dilute" conducting subbands as those in Ref 9 and 10 , but the structure in these subbands exhibits some kind of self-similarity and become more complicated when the stage of the system increases. As a result, the calculated conductance sensitively depends on the structure parameters $(m, n)$ of the original hierarchical fractal and on the position of the Fermi level.

The paper is organized as follows: In the next section we present the derivation of the equivalent 1D model and the basic formalism. In Section III the calculated transmission spectrum and conductance are illustrated. Section IV is devoted to a brief summary and discussion.

\section{BASIC FORMALISM}

According to Samukhin et. al, the hierarchical model for the stretched polymer is constructed in the following way: as a first stage, $n$ primitive bonds are taken to form an $n$-length chain along stretched direction, and $m$ threads of such chains are combined at both ends to form an $(m, n)$ boundle with two common terminals; in the following stages the boundles formed in the previous stage are taken as the elementary bonds and the same procedure is performed to form new boundles. We are interested in the variation of the transmission spectrum in increasing the number of stages $N$, i.e. the size of the system. In measuring the conductance one needs to link electrodes to both ends, (see Fig.1). We use two integers, $(i, j)$, to label the sites, where $i$ indicates the position in the longitudinal direction and 
$j$ in the transverse direction. If the number of stages of the system is $N$, the longitudinal index $i$ is varying in the range of $\left[0, n^{N}\right]$. For a given $i$, the number of sites in the transverse direction is

$$
\rho_{N}(i)=\left\{\begin{array}{l}
m^{N}, \text { if } \bmod (i, n) \neq 0 \\
m^{N-\alpha_{N}(i)} \text { if } \bmod \left(i, n^{\alpha_{N}(i)}\right)=0 \text { and } \bmod \left(i, n^{\alpha_{N}(i)+1}\right) \neq 0, \alpha_{N}(i) \text { is integer in }(0, N) ; \\
1, \text { if } i \leq 0 \text { or } i \geq n^{N}
\end{array}\right.
$$

where $\bmod (x, y)$ denotes the remainder on the division of $x$ by $y$. Thus, in the site index $(i, j), j$ is ranging in $\left[1, \rho_{N}(i)\right]$. From the tight binding approach, the Schrödinger equation can be written as

$$
E \psi_{i, j}=\epsilon_{i, j} \psi_{i, j}+\sum_{\delta(i, j)} t_{i j, \delta(i, j)} \psi_{\delta(i, j)}
$$

where $\psi_{i, j}$ is the coefficient of the wave function on site $(i, j), \delta(i, j)$ denotes the position of next neighbors of $(i, j)$, $\epsilon_{i, j}$ is its site energy, $t_{i j, \delta(i, j)}$ the hopping between sites $(i, j)$ and $\delta(i, j)$. As we are only interested in the topological structure of the system, we can assume that the site energies and the nearest-neighbor hoppings are independent of the position. In this case one can set the site energy to be the energy origin and the hopping to be the energy unit. By summing over the transverse sites for a given $i$, Eq. (2) becomes

$$
E \psi_{i}=\beta_{N}(i, i+1) \psi_{i+1}+\beta_{N}(i, i-1) \psi_{i-1}
$$

where

$$
\psi_{i}=\frac{1}{\sqrt{\rho_{N}(i)}} \sum_{j=1}^{\rho_{N}(i)} \psi_{i, j}
$$

and

$$
\beta_{N}\left(i, i^{\prime}\right)=\max \left(\sqrt{\frac{\rho_{N}(i)}{\rho_{N}\left(i^{\prime}\right)}}, \sqrt{\frac{\rho_{N}\left(i^{\prime}\right)}{\rho_{N}(i)}}\right) .
$$

From Eq. (1) it is easy to see that

$$
\beta_{N}\left(i, i^{\prime}\right)=m^{\left|\alpha_{N}(i)-\alpha_{N}\left(i^{\prime}\right)\right| / 2},
$$

where $\alpha_{N}(i)$ is the largest power of factor $n^{\alpha_{N}(i)}$ of integer $i$ in the range $\left[1, n^{N}-1\right]$, as defined in Eq. (11), and we set $\alpha_{N}(i)=N$ for $i \leq 0$ and $i \geq n^{N}$.

From Eq. (3) one can see that the fractal structure reduces to a 1D effective Hamiltonian with special aperiodic structure. In the derivation we have adopted the summation over the transverse sites which erases the degree of freedom of electrons in this direction. This has no effect on the longitudinal transport properties for the one-channel incoming and outgoing leads of the present geometry. The situation becomes different if multichannels are connected to the system or there exists on-site or off-diagonal disorder in the system which can produce an energy-dependent terms in the reduced Hamiltonian. In this paper we only focus on the present geometry and the multichannel and disordered cases will be considered in separated ones.

The transmission coefficient in such a one-channel structure can be calculated by the standard transfer matrix technique. The transfer matrix can be defined as follows

$$
\begin{gathered}
\left(\begin{array}{c}
\psi_{i+1} \\
\psi_{i}
\end{array}\right)=\left(\begin{array}{cc}
E m^{-\frac{\left|\alpha_{N}(i)-\alpha_{N}(i+1)\right|}{2}} & -m^{\frac{\left|\alpha_{N}(i)-\alpha_{N}(i-1)\right|-\left|\alpha_{N}(i)-\alpha_{N}(i+1)\right|}{2}} \\
1 & 0
\end{array}\right)\left(\begin{array}{c}
\psi_{i} \\
\psi_{i-1}
\end{array}\right) \\
\equiv B_{N}(i)\left(\begin{array}{c}
\psi_{i} \\
\psi_{i-1}
\end{array}\right) .
\end{gathered}
$$

So along the $1 \mathrm{D}$ chain with stage number $N$, the wave function of an electron in two leads are related to each other via a total transfer matrix

$$
\left(\begin{array}{c}
\psi_{n^{N}+1} \\
\psi_{n^{N}}
\end{array}\right)=\prod_{i=0}^{n^{N}} B_{N}\left(n^{N}-i\right)\left(\begin{array}{c}
\psi_{0} \\
\psi_{-1}
\end{array}\right) \equiv \mathbf{T}(N)\left(\begin{array}{c}
\psi_{0} \\
\psi_{-1}
\end{array}\right)
$$


We demonstrate such an effective 1D chain structure in Fig. 2. This is a one-to-one mapping from the original network of Fig.1, and the resultant 1D chain shares the hierarchical structure at its longitudinal direction and the mirror reflecting symmetry as well. The self-similar features of the original structure are also kept in the mapping.

The $\rho_{N}(i)$-structure in the longitudinal direction of the original system can be described by the following series:

$$
1, \underbrace{m^{N}, \cdots, m^{N}}_{n-1}, \underbrace{m^{N-1}, \underbrace{m^{N}, \cdots, m^{N}}_{n-1}, \cdots, m^{N}, \underbrace{m^{N-1}, \cdots, m^{N}}_{n-1}}_{n-1}, m^{N-2}, \cdots
$$

One can see that this is a embedded multi-period structure. As a consequence the hoppings in the effective 1D chain are arranged as in the following series:

$$
1, m^{\frac{N}{2}} \underbrace{1, \cdots, 1}_{n-2}, m^{\frac{1}{2}}, \underbrace{m^{\frac{1}{2}}, \underbrace{1, \cdots, 1}_{n-2}, m^{\frac{1}{2}}, \cdots, m^{\frac{1}{2}}, \underbrace{1, \cdots, 1}_{n-2}, m^{\frac{1}{2}}}_{n-2}, m^{\frac{1}{2}}, \underbrace{1, \cdots, 1}_{n-2}, m^{\frac{2}{2}}, \cdots .
$$

Thus, one has the following recursion relation of the transfer matrix for $n \geq 2$ :

$$
\begin{gathered}
\mathbf{T}(N)=B_{N}\left(n^{N}\right) B_{N}\left(n^{N}-1\right) B_{N-1}\left(n^{N-1}-1\right) \mathbf{C}(N-1)[\mathbf{C}(N-1) \\
\times \mathbf{F}(N-1)]^{n-2} \mathbf{C}(N-1) B_{N-1}^{-1}(1) B_{N}(1) B_{N}(0),
\end{gathered}
$$

where

$$
\mathbf{C}(N-1)=B_{N-1}^{-1}\left(n^{N-1}\right) \mathbf{T}(N-1) B_{N-1}^{-1}(0),
$$

and

$$
\mathbf{F}(N-1)=\left(\begin{array}{cc}
\frac{E}{\sqrt{m^{N-1}}} & -1 \\
1 & 0
\end{array}\right)
$$

One can find that the effective $1 \mathrm{D}$ chain contains $n^{N}$ bonds among which there are $(n-2) n^{N-1}$ bonds of hopping 1, 2 bonds of hopping $\sqrt{m^{N}}$, and $2 n^{N-i-1}(n-1)$ bonds of hoppings $\sqrt{m^{i}}$ for $i=1,2, \cdots, N-1$. These bonds are arranged in a special aperiodic way so that one may expect a self-similar and Cantor-set-like transmission spectrum. From the transfer matrix $\mathbf{T}$, we can easily calculate the transmission coefficient for electrons from the left to the right lead:

$$
\tau(E)=\frac{4 \sin ^{2} k}{\left[T_{21}-T_{12}+\left(T_{22}-T_{11}\right) \cos k\right]^{2}+\left(T_{11}+T_{22}\right)^{2} \sin ^{2} k},
$$

where $k=\arccos (E / 2)$ is the wave vector of wave function in the leads. Thus, the conductance of the original network at the longitudinal direction is obtained according to Landauer formula

$$
g(E)=\frac{e^{2}}{h} \int \tau(E) \frac{\partial f}{\partial E} d E
$$

where $f$ is the Fermi distribution function

$$
f(E, T)=\frac{1}{1+\exp \left(E-E_{f}\right) / k_{B} T} .
$$

\section{TRANSMISSION SPECTRUM AND TEMPERATURE DEPENDENCE OF CONDUCTANCE}

By using the above formulas we calculate the transmission coefficient as a function of energy for different structure parameters $(m, n)$ and different stage number. Typical results of transmission are illustrated in Fig. 3 and Fig. 4. We can see that when the stage number of the structure is low (say $N \leq 2), \tau(E)$ exhibits quite continuous variation with broad peaks and dips. However, when the stage number $N \geq \overline{3}$ the whole energy interval [-2,2] is divided into "subbands" which allow electron transmission and "gaps" of nearly zero transmission with sharp edges. 
By increasing $N$, the subbands are further divided into "minibands" and "minigaps", but the edges of the main subbands are almost independent of $N$. For large $N$, there are a large number of sparsely distributed peaks with $\tau(E)$ equal to or near unity which indicates almost complete transition corresponding to unscattered electronic states. This structure of the transmission spectrum also shows the self-similarity and Cantor-set-like behavior, like the situation in the Fibbonaci series and other quasiperiodic or aperiodic systems. By the comparison between Fig. 3 and Fig. 4, we find that although the number of the main subbands is the same but the width of the central main subband is slightly widened and the other two are shifted away from the band center by increasing $n$, which changes the number of the repeated bonds in the series. In the inset of Fig. 1(a) we also show the $N$ dependence of the transmission coefficient for energy $E=1.57$ which lies in the main band. It can be seen that for this energy by increasing $N$ the transmission first decreases but then increases. This behavior is sensitive to the position of $E$ as can be seen from the complicated structure in the transmission spectrum for large $N$.

For the polymer networks, an interesting problem is to investigate the scaling behavior and the temperature dependence of the dc conductance. Since the transmission subbands exhibit "dilute" characteristics with a lot of transmission holes within them, the temperature dependence of the conductance is sensitive to the position of the Fermi level. From Eq. (11), for low temperature the conductance as a function of the Fermi level shows almost the same characteristics as those for the transmission coefficient as a function of energy. From Figs. 3 and 4 one can see that there exist energy points for which the transmission of large system is greater than that of small system. As a consequence for the system with Fermi level at these points the conductance increases with the system size provided that the temperature is low enough. This may be regarded as the "metallic" scaling behavior. There are still many other energy points for which the scaling behavior of the conductance is "insulating" as it decreases with the system size. However, the energy regimes for "metallic" and "insulating" states are strongly mixed with each other and form a complicated Cantor-set-like structure. If the temperature becomes higher, such a structure is smoothened by the thermal averaging. In Figs. 5 and 6 we plot the Fermi level dependence of the conductance at temperature $T=400 \mathrm{~K}$ for different structures and different system size. It can be seen that at this temperature the curves become smooth and in the whole energy range the conductance decreases with the increase of the system size, showing the insulating behavior. This is because more transmission holes appear in the transmission subbands when $N$ increases and this reduces the average of the transmission. In Fig. 7 we plot the temperature dependence of conductance $g(T)$ for one structure but with different Fermi levels. The curves manifest that the conductance can increase or decrease with the decrease of the temperature, strongly depending on the Fermi level $E_{f}$. The orders of magnitude of the variation of conductance at the range of $[50 \mathrm{~K}, 400 \mathrm{~K}]$ could vary from 1 to 4 . Only for carefully chosen values of $E_{f}$ (usually near edges of subbands for a gizen structure $(m, n)$ ), one could get a curve of $\log g(T)-T^{-1 / 2}$ whose slope can be fitted to the experimental datall 1 . In fact, the realistic stretched polymers should contain tremendous number of such networks in different stages, and doping might adjust Fermi level as well as the distribution of $(m, n)$. This may cause the deviation of the conductance calculated results in this simple model from the observed data. Nevertheless, as one can see from Fig. 7, our calculation displays a curve of good fitting to expression $\ln g_{d c} \propto T^{-1 / 2}$ within quite large range of temperature, while the solution of the model from the critical percolation approach in Ref. 0 demonstrates that $g_{d c} \propto \frac{e^{-\left(T_{0} / T\right)^{1 / 2}}}{T^{2}}$ which slightly diverges from experimental results.

\section{CONCLUSIONS AND DISCUSSION}

In summary, we have shown that the hierarchical fractal network of Fig. 1 is equivalent to a $1 \mathrm{D}$ aperiodic series if only one-channel transport properties are under consideration. This equivalence is derived by summing over the transverse degree of freedom and, as a consequence, does not imply the one-dimensionality of this structure. The self-similarity and the aperiodicity of the original network are kept in this mapping. We calculate the transmission spectrum and temperature dependence of the conductance for systems with different structure parameters and various size. The transmission spectrum manifests Cantor-set-like characteristics, with complicated structures made of mixed transmission peaks and gaps, like most of the 1D quasiperiodic and aperiodic systems. Such a structure is also reflected in the mixed insulating and metallic scaling behavior of the conductance at low temperature. At higher temperature such a structure in the conductance is smoothened by the thermal averaging and the scaling behavior is insulator like in the whole energy range. This implies some type of metal-insulator transition at special values of the Fermi level, in agreement with the analysis in Ref 11 . The magnitude and temperature dependence of the conductance strongly depend on the position of the Fermi level. By adjusting the value of $E_{f}$, one can reproduce the temperature dependence observed in the experiments. This simplified model can catch several features of polymer qualitatively but a complete description needs more sophisticated models. 


\section{ACKNOWLEDGMENTS}

This work is supported by National Fund of Natural Science of China.

${ }^{1}$ Zhao H. Wang and Hamid H. S. Javadi, Phys. Rev. B 42, 5411 (1990).

${ }^{2}$ J. Joo, Oblakowski, and G. Du, Phys. Rev. B 49, 2977 (1994).

${ }^{3}$ N. Basescu, Z -X. Liu, D. Moses, A. J. Heeger, H. Narrmann, and Theophilou, Nature (London) 327, 403 (1987).

${ }^{4}$ J. Tsukamoto, A. Takahasi, and K. Kawasaki, Jpn. J. Appl. Phys. 29, 125 (1990).

${ }^{5}$ Y. Nogami, H. Kaneko, T. Ishiguro, N. Hosoito, A. Takahashi, and J. Tsukamoto, Solid State Commun. 76, 583 (1990).

${ }^{6}$ Y. Nogami, H. Kaneko, H. Ito, T. Ishiguro, T. Sasaki, N. Toyota, A, Takahashi, and J. Tsukamoto, Phys. Rev. B 43, 11829 (1991).

${ }^{7}$ H. H. Javadi, A. Chakraborty, C. Li, N. Theophilou, D. B. Swanson, A. G. MacDiarmid, and A. J. Epstein, Phys. Rev. B 43, 2183 (1991).

8 J. Joo, V. N. Prigodin, Y. G. Min, A. G. MacDiarmid and A. J. Epstein, Phys. Rev. B 50, 12226 (1994).

${ }^{9}$ Shi-Jie Xiong and S. N. Evangelou, Phys. Rev. B 52, R13079 (1995).

${ }^{10}$ Shi-Jie Xiong, Yan Chen, and S.N. Evangelou, Phys. Rev. Lett. 77, 4414 (1996).

11 A. N. Samukhin, V. N. Prigodin, and L. Jastrabik, Phys. Rev. Lett. 78, 326 (1997).

12 X -S. Chen and S -J. Xiong, Phys. Lett. A 179, 217 (1993).

FIG. 1. Hierarchical fractal network with structure parameters $m=3, n=4$ and stage number (a) $N=1,(\mathrm{~b}) N=2$.

FIG. 2. The equivalent 1D chain for the hierarchical structure shown in Fig. 1 with the same parameters. The numbers below the lines indicate the index of sites in the longitudinal direction, and the numbers above the lines are the effective hopping integral of the corresponding bonds.

FIG. 3. The transmission coefficient as a function of energy for structures with $m=2, n=5$, and (a) $N=1$, (b) $N=2$, (c) $N=3,($ d) $N=4$, (e) $N=5$. The inset of (a) shows the $N$ dependence of $\tau(E)$ for $E=1.57$.

FIG. 4. The transmission coefficient as a function of energy for structures with $m=2, n=6$, and (a) $N=1$, (b) $N=2$, (c) $N=3,($ d) $N=4$.

FIG. 5. The conductance as a function of Fermi level at temperature $T=400 \mathrm{~K}$ for structures with $m=2, n=5$, and (a) $N=1$, (b) $N=2$, (c) $N=3$, (d) $N=4$, (e) $N=5$. The hopping integral of the original network is set to be $1 \mathrm{eV}$.

FIG. 6. The conductance as a function of Fermi level at temperature $T=400 \mathrm{~K}$ for structures with $m=2, n=6$, and (a) $N=1$, (b) $N=2$, (c) $N=3$, (d) $N=4$. The hopping integral of the original network is set to be $1 \mathrm{eV}$.

FIG. 7. The conductance as a function of temperature of system with structure parameters $m=2, n=5$ and $N=5$ for different Fermi levels. 

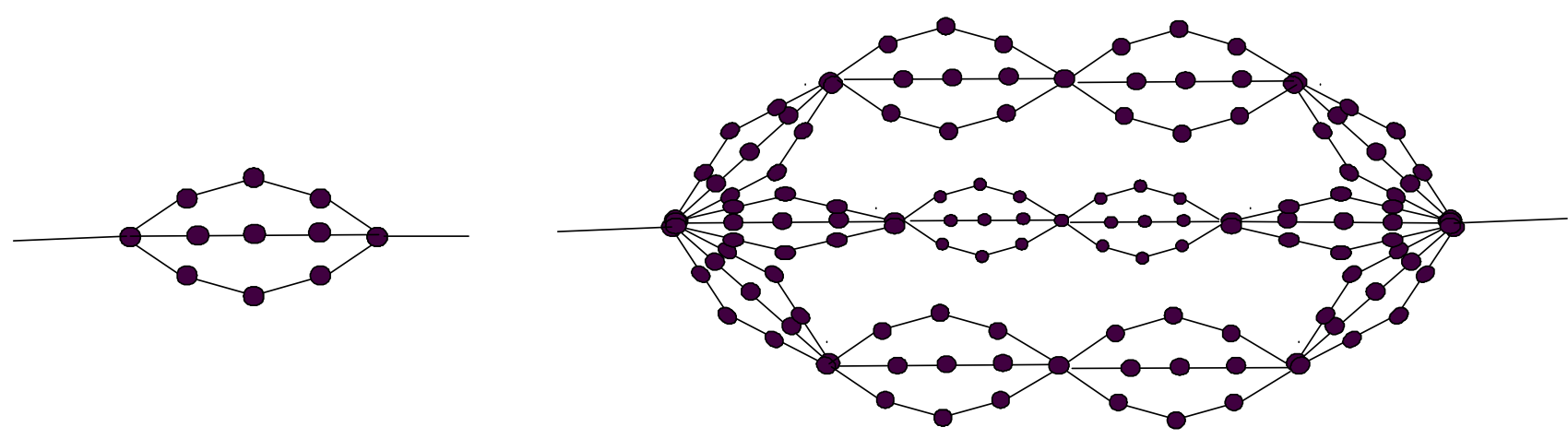

(a)

(b)

Fig. 1 
Fig. 2

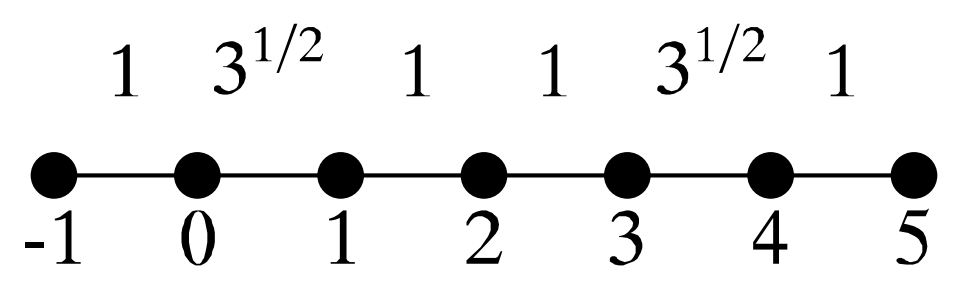

(a)

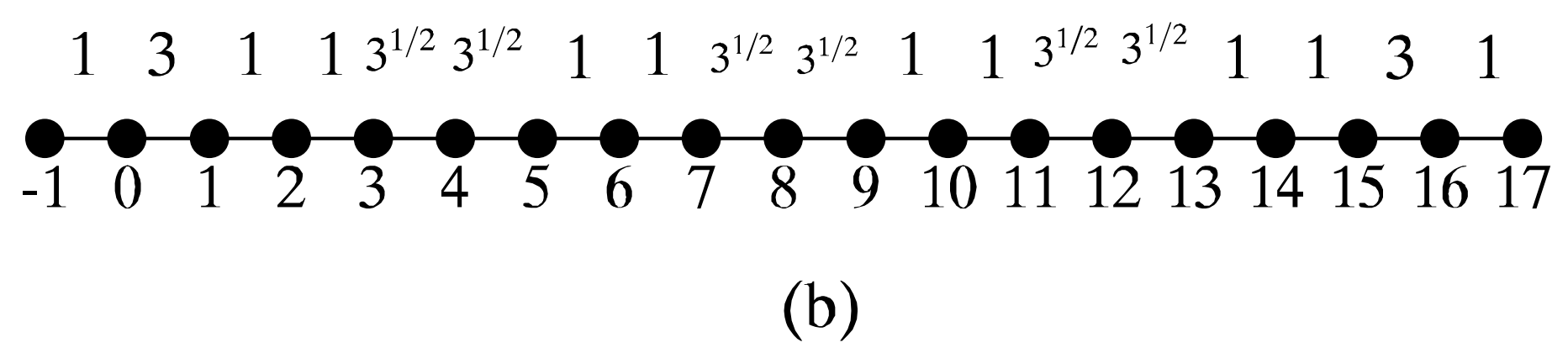


$m$

으

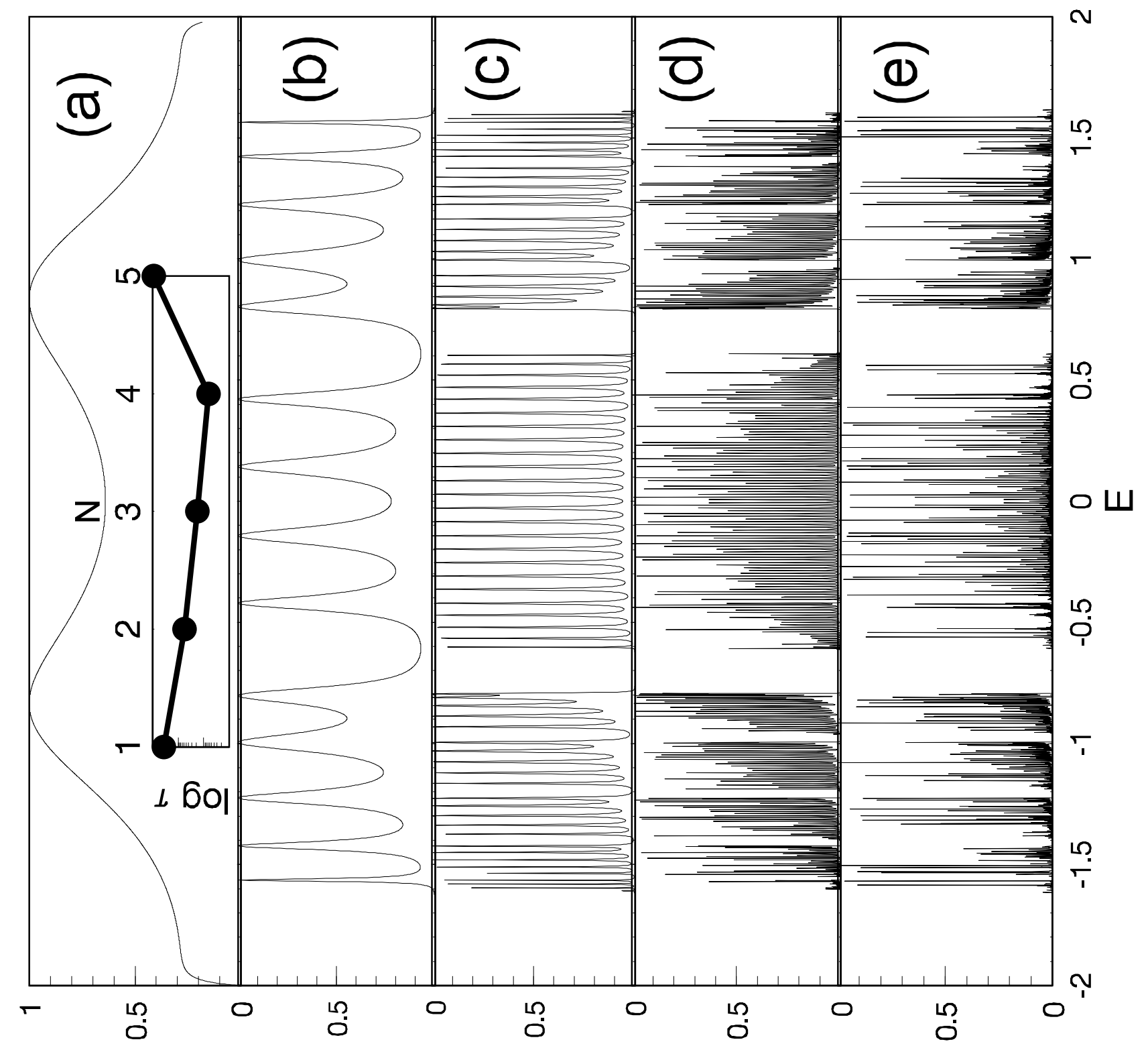

(ヨ) \llcorner 


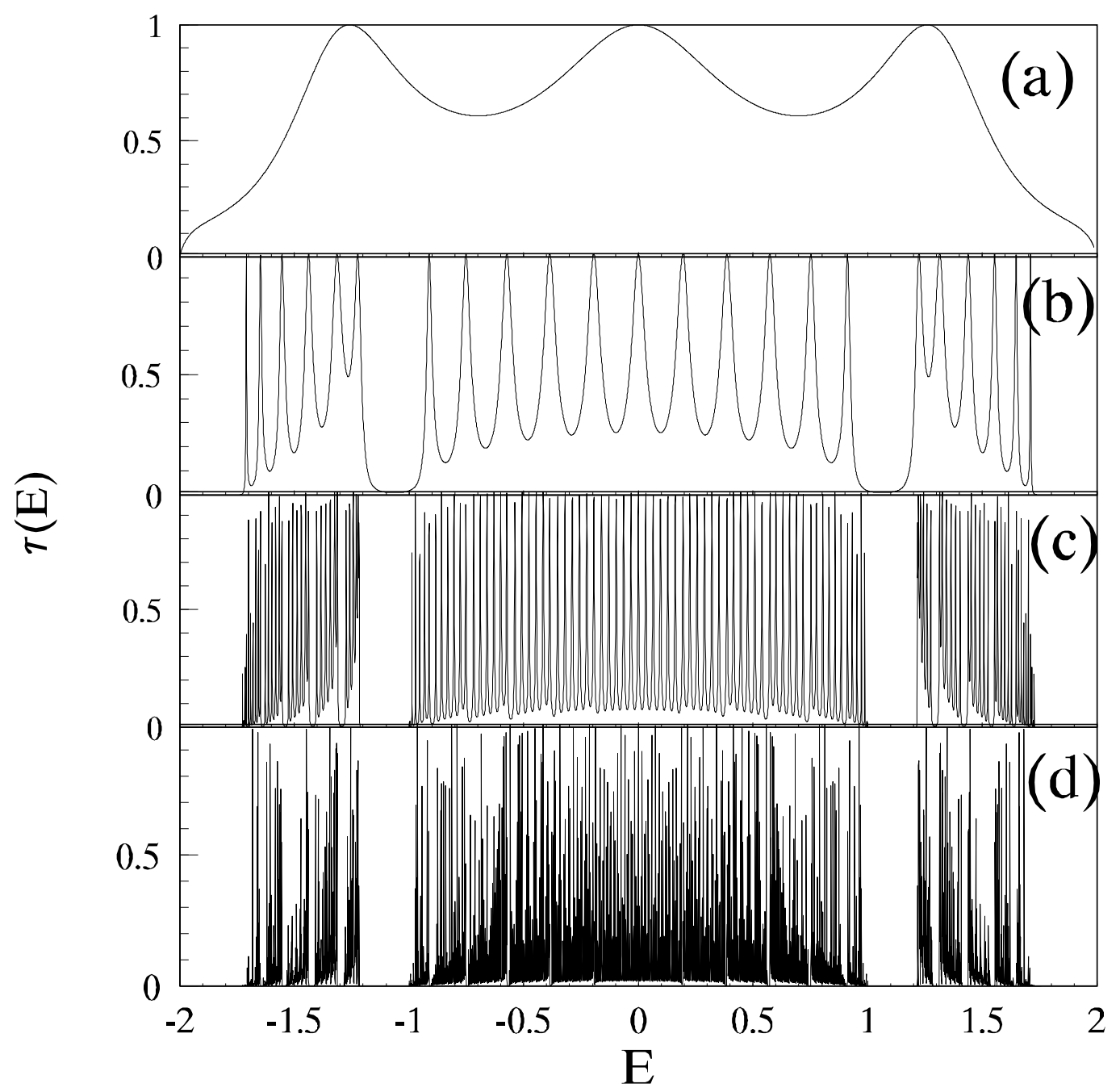

Fig. 4 
ก

음

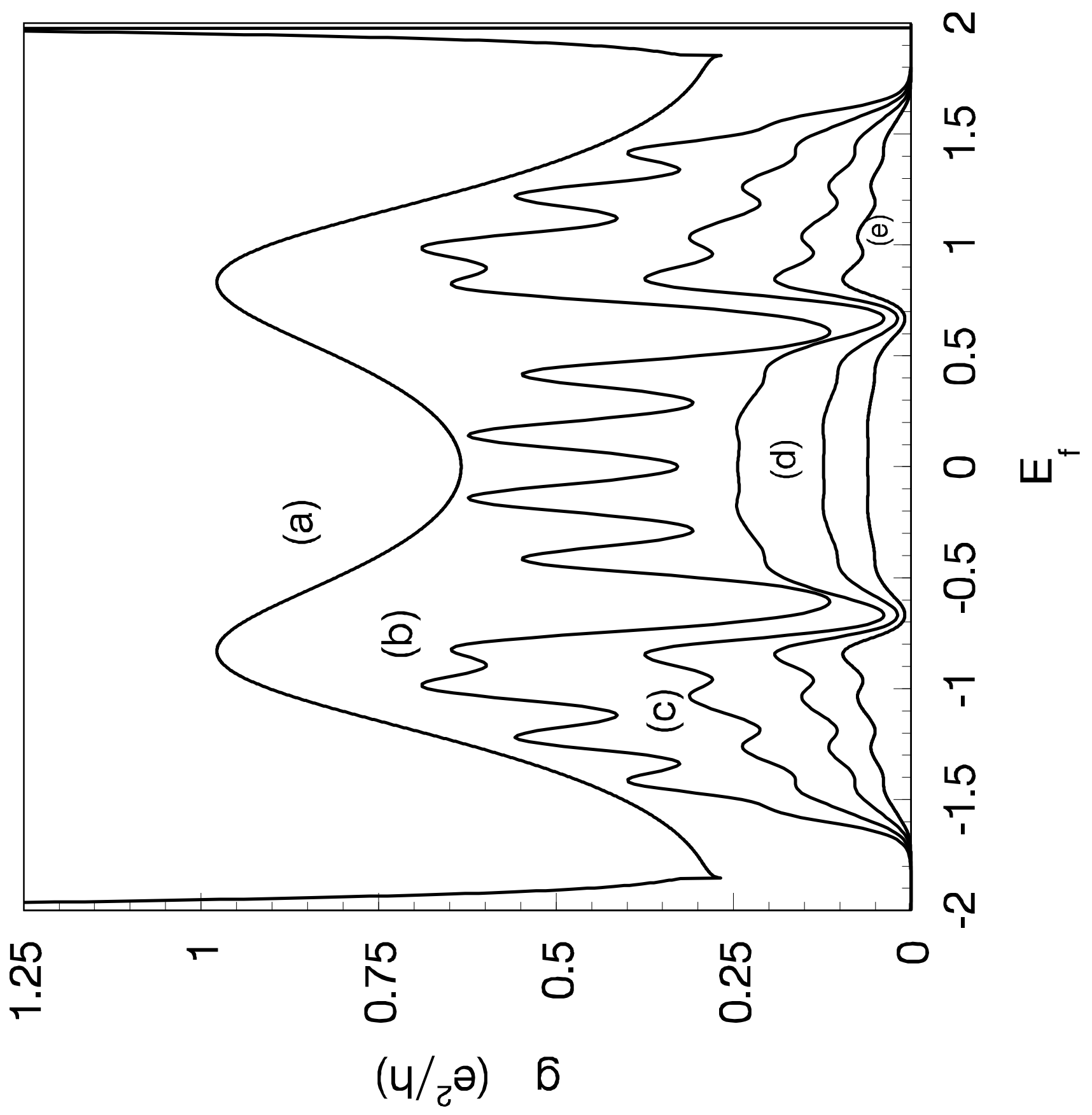


6

문

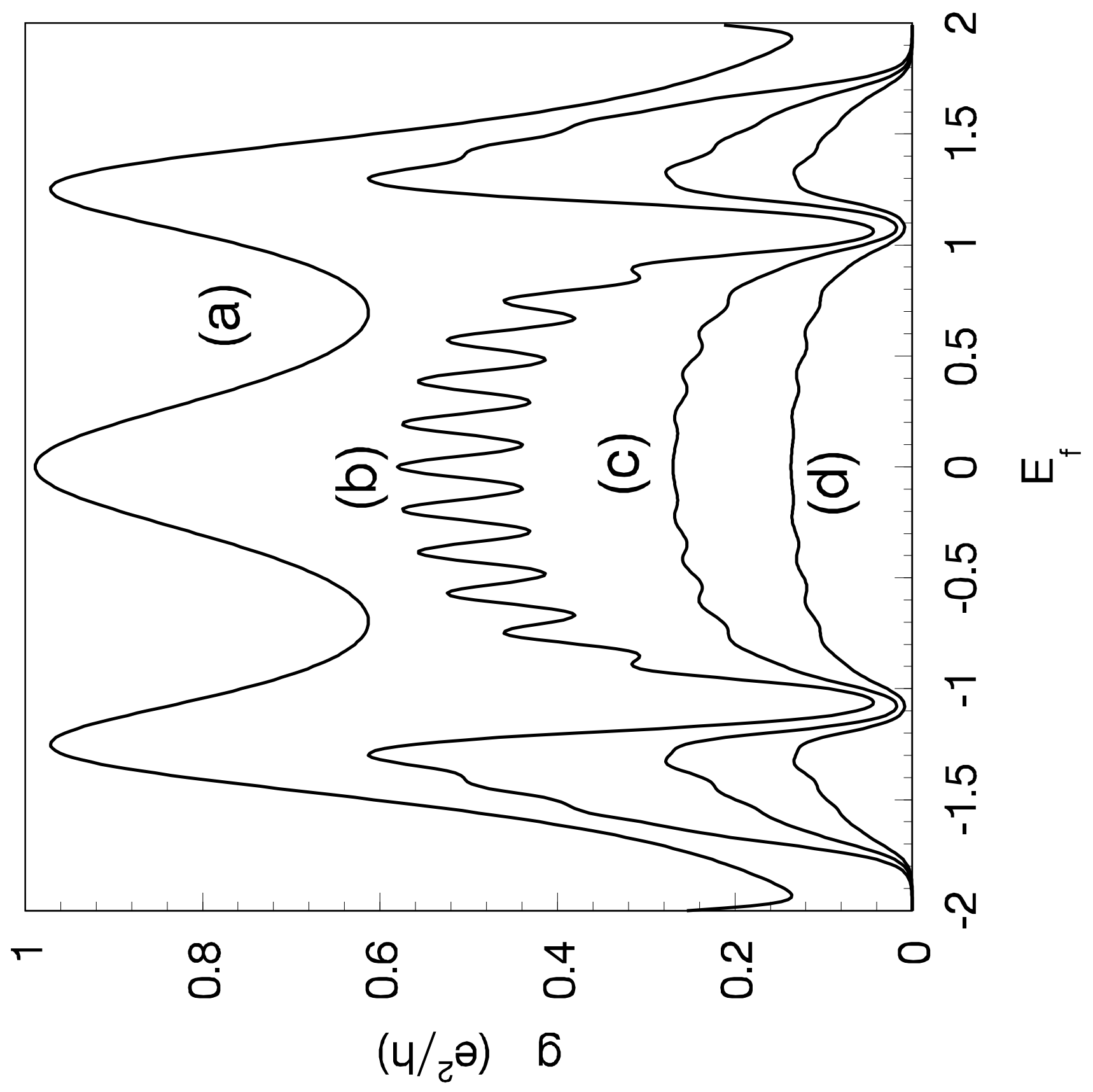




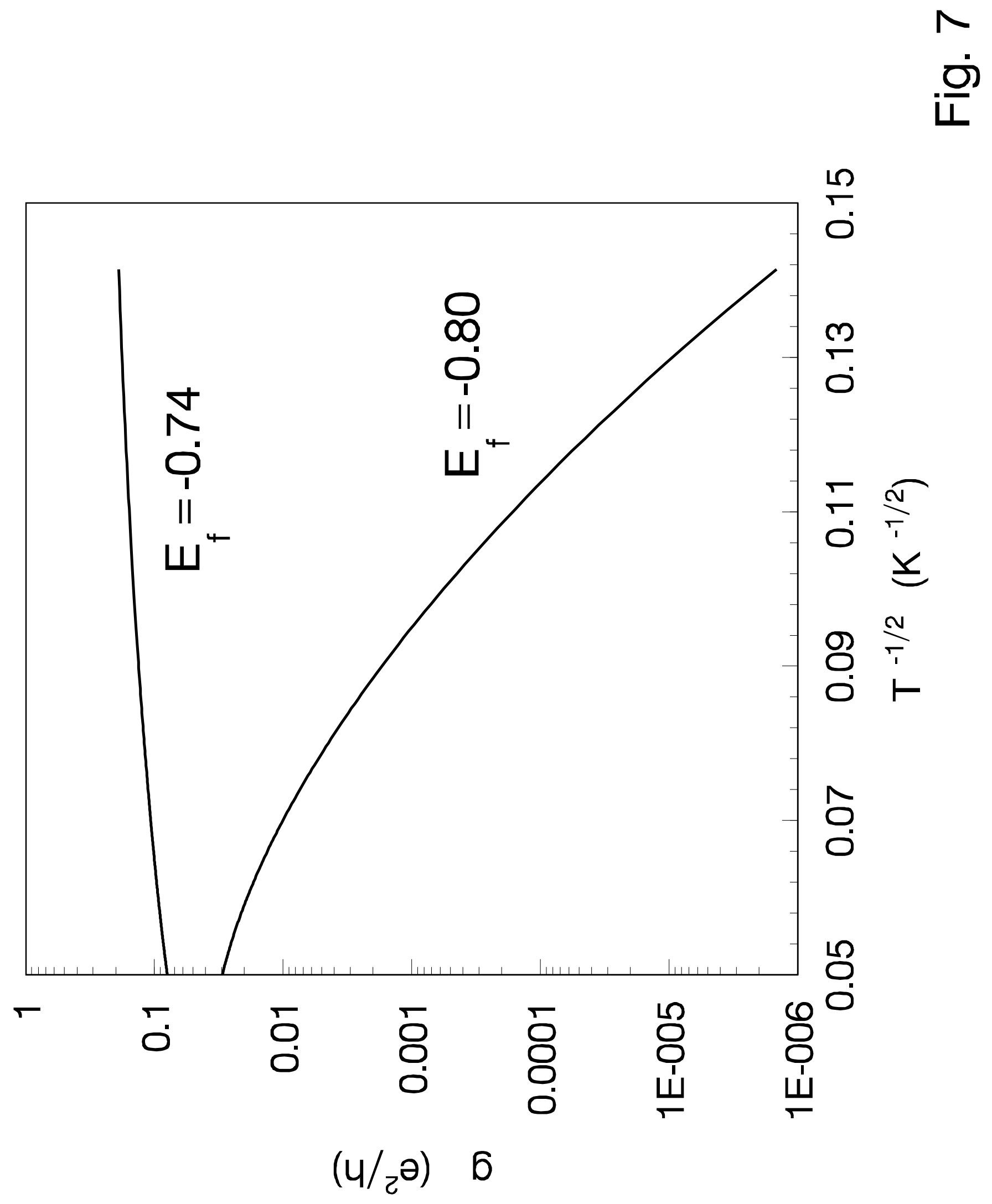

